\title{
Data-driven Applications to Foster Absorptive Capacity: A Literature-based Conceptualization
}

\author{
Peter Hofmann \\ Project Group Business \& Information Systems \\ Engineering of the Fraunhofer FIT, \\ FIM Research Center, University of Bayreuth \\ peter.hofmann@fim-rc.de \\ Christoph Buck \\ Centre for Future Enterprise, QUT Business School, \\ Queensland University of Technology \\ christoph.buck@qut.edu.au \\ Philipps-Universität Marburg \\ christoph.buck@wiwi.uni-marburg.de
}

\begin{abstract}
The relevance of data-driven applications for leveraging knowledge embedded in data is growing. Thereby, an organization's capability to create, disseminate, and exploit knowledge (i.e., absorptive capacity) is a decisive factor in gaining competitive advantages. In this paper, we address the lack of guidance on the development and application of datadriven applications fostering an organization's absorptive capacity. Based on a structured literature review, we derive seven data-driven application capabilities and match them with an established conceptualization of absorptive capacity. While previous literature did not allow for a specific analysis, our functional representation concretely demonstrates how data-driven applications composed of separate capabilities can foster absorptive capacity in manifold ways. This paper contributes to the literature by providing a structured literature review on the impact of IT on absorptive capacity as well as introducing theoretically-based and modularized data-driven application capabilities.
\end{abstract}

\section{Introduction}

Knowledge guides decision-making and improves business processes as well as outcomes and, consequently, allows a company to gain competitive advantages [19]. Therefore, a company's capability to create, disseminate, and exploit knowledge is a decisive success factor [16, 41]. Thereby, relevant knowledge exists both within an organization and in its environment [58], and knowledge can be encapsulated in data [19]. Hence, advancements in

\author{
Philipp Stähle \\ University of Bayreuth \\ Philipp.staehle@uni-bayreuth.de
}

Harald Thorwarth

University of Applied Forest Sciences Rottenburg thorwarth@hs-rottenburg.de data processing methods and the exponentially growing availability of knowledge, embedded in data, can be leveraged by organizations. By implementing a data-driven application (DDA), companies can not only process large datasets efficiently but support decision-making effectively [40]. In our paper, we understand a DDA as a software application within an information system that supports leveraging data's or information's business potential. In this context, we understand an information system as "a system in which human participants and/or machines perform work (processes and activities) using information, technology, and other resources to produce informational products and/or services for internal or external customers" [4, p. 451]. However, effectively leveraging knowledge's potential is a challenging endeavor [e.g., 34, 45]. Even if DDAs expose knowledge hidden in data, the mere exposure of knowledge is not necessarily concomitant with knowledge's beneficial use [43]. Knowledge may exist in isolated organizational silos [33], leading to the challenge of distributing knowledge across the organization [28]. Hence, companies require a sociotechnical understanding of how DDAs can foster the beneficial use of knowledge gained from data. Without this understanding, companies are flying blind, and risk developing and applying DDA that waste resources.

By taking a comprehensive perspective, absorptive capacity (AC) is a promising theoretical lens to explain an organization's ability to identify, assimilate, transform, and apply knowledge to gain competitive advantages [10, 59]. Information Systems (IS) literature has already discussed IT capabilities fostering AC [e.g., 22, 29, 46], but very abstractly [46]. 
Moreover, existing research on $\mathrm{AC}$ often does not provide sufficient differentiation between data, information, and knowledge in order to explain DDAs' comparative functionality in detail. Thus, existing research lacks a systematic elaboration of DDAs' effect on AC. To close this research gap, we pose the research question: What are the capabilities of DDAs that foster an organization's AC?

To address the research question, we took a threephase approach that bases on the insights of a structured literature review consisting of two literature searches. For conducting the literature review, we follow Webster and Watson [55]. In the first phase, we investigate IT capabilities discussed in the AC discourse. In the second phase, we include the identified knowledge management literature to compare and harmonize concepts. This allows us to ultimately derive comprehensive DDA capabilities that incorporate insights from both the $\mathrm{AC}$ and knowledge management discourse. In the third phase, we match the DDAs with the established conceptualization of the AC dimensions introduced by Zahra and George [59].

\section{Theoretical foundations}

AC is the ability to create new collective knowledge from external knowledge toward beneficial use to products and services [10]. Hence, we follow the predominant theoretical view and regard $\mathrm{AC}$ as an organizational capability in our paper [46]. Among others, $\mathrm{AC}$ is an enabler for business success in turbulent environments [31], especially in information-intensive environments [36]. Additionally, AC is a construct of the knowledgebased view $[36,46]$. The knowledge-based view is an extension of the resource-based view and a field in strategic management theory against the backdrop of the information age. At its core, the knowledge-based view considers knowledge and capabilities as the essential strategic resources of an organization and investigates the problems of knowledge coordination [18]. Thus, knowledge and capabilities are a source from which to improve value creation and competitive advantage [31, 46]. Several IS scholars have developed frameworks or models to better capture AC [e.g., 28, 43, 46, 59]. Among others, Zahra and George [59] provide an established, clear, and operationalized model of AC. They define four dimensions of AC, namely: acquisition, assimilation, transformation, and exploitation. Acquisition refers to the ability to identify and acquire new knowledge (e.g., monitoring customer demand). Assimilation is the ability to comprehend externally identified knowledge by analysis and interpretation (e.g., interpreting whether change in customer demand matters for a company and its products or not). Transformation describes the ability to refine the routines that enable the combination of newly assimilated and existing knowledge (e.g., evaluating whether the company needs to adjust routines to react to changing customer demand). Exploitation is the ability to use the transformed knowledge for commercial purposes (e.g., adjusting or creating new products satisfying a changed customer demand). Additionally, Zahra and George [59] distinguish potential and realized AC. While potential AC comprises the acquisition and assimilation dimensions, realized $\mathrm{AC}$ comprises the transformation and exploitation dimensions [59].

Similar to AC, the research on knowledge management deems identifying and managing knowledge effectively as a source of competitive advantage in organizations [e.g., 14]. Following Liebowitz [33], we understand knowledge management as the process of leveraging knowledge internally in the organization and externally to the customers and stakeholders to create value [33]. Knowledge management's activities overlap with activities that are commonly associated with AC, despite originating from different academic discourses. Existing research yields a cluster of different approaches to structuring knowledge management's activities or processes. Heisig [24] emphasizes the five most frequently mentioned activities within 160 knowledge management frameworks: to share, create, apply, store, and identify.

Despite overlapping conceptually, the literature on knowledge management is more focused on IT than the literature on AC since scholars consider IT capabilities as a decisive factor for implementing or supporting knowledge management in organizations [e.g., 3]. Some scholars even refer specifically to knowledge management systems as "IT-based systems developed to support and enhance the organizational processes of knowledge creation, storage/retrieval, transfer, and application" [3]. For instance, research on knowledge management covers DDAs such as decision support systems [40], knowledge-based systems [2], expert systems [15], or data mining [53].

\section{Research method}

With this paper, we aim to shed light on how DDAs foster an organization's AC. To answer our research question, we conducted a structured literature review consisting of two search strategies following Webster and Watson [55] and took a three-phase approach as illustrated in Figure 1. 


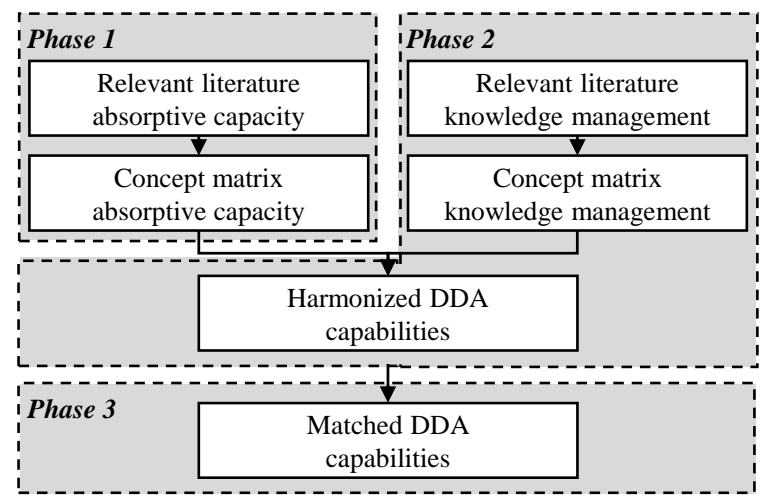

Figure 1: Research approach

In the first phase, we aimed to identify and analyze IT capabilities discussed in the body of AC literature and, thus, investigate the current state of research regarding the impact of IT capabilities on AC (see Section 4). To identify relevant literature, we relied on the following search string: 'absorptive capacity AND capabilities AND (information technology OR information system)'. We searched from March to April 2020 in the databases Web of Science, AISel, and EBSCO Business Source Premier and restricted the results to English language journal articles. The initial sample - duplicates excluded - included 351 search results. We deemed papers as relevant if they completely or partly focus on the relation between IT and AC. We excluded studies that do not address the subject at all or that do not bridge the gap between IT and $\mathrm{AC}$ or a related subject. Thus, we did not consider papers focusing only on AC (or a related subject) but not on IT and vice versa. After scanning the remaining papers' titles and abstracts, 34 papers remained. We then conducted a full-text screening of the remaining papers, resulting in 18 relevant papers.

In the second phase, we aimed to identify DDA capabilities fostering AC (see Section 5). Therefore, we conducted a second literature search from April to May 2020 covering the knowledge management discourse to broaden the perspective. The knowledge management discourse allowed us to introduce a new and, above all, more technical perspective. The second literature search relied on the following search string: 'knowledge management' AND ('information technology' OR 'information system' OR 'datadriven') AND ('activity' OR 'process' OR 'function' OR 'framework')'. As in the first literature search, we searched in the databases Web of Science, AISel, and EBSCO Business Source Premier and restricted the results to English language journal articles. The initial sample - duplicates excluded - included 872 search results. We considered papers as relevant if they discuss whether or how one or more DDAs affect knowledge management activities. We excluded papers for two reasons: First, the paper does not consider one or more DDAs. Hence, the examined application must create business potential by producing informational products and/or services from data or information. Second, papers must not miss making the link between a technology and a knowledge management activity. In the second literature search, we got 262 papers after screening the papers' titles and abstracts, and 30 relevant papers after the full-text screening. In order to derive specific DDA capabilities, we combined both literature searches, resulting in a total of 48 relevant papers for the second phase of our research. After identifying the relevant literature in both domains (i.e., $\mathrm{AC}$ and knowledge management), we separately developed a concept matrix for the literature body of each domain. We then compared the results to harmonize concepts. Within concept harmonization, we merged similar concepts or extended concepts by incorporating further aspects. To do so, we iteratively developed and discussed the DDA capabilities in the research team. To illustrate the results, we have enriched the capabilities with examples that are not necessarily part of the results of the structured literature search.

In the third phase, we mapped the DDA capabilities to the AC dimensions by relying on the underlying literature (see Section 6). To do so, we referred to the AC dimensions of Zahra and George [59] as described in Section 2.

\section{IT capabilities for AC}

Prior IS research regards IT as an enabler for the collection, storage, and dissemination of organizational knowledge [28]. Moreover, IT is assumed to play an important role in enabling knowledge transfer [3, 48]. Additionally, some scholars attribute to IT an increasingly direct effect on AC $[28,43,46]$. We summarize existing research on the relationship between IT and AC in Table 1. In the column scope, we specify the different levels of IT capabilities. The last four columns cover the mapping of the examined IT capabilities to the AC dimensions acquisition (aq), assimilation (as), transformation (tr), and exploitation (ex) according to Zahra and George [59].

Comparing the scope of IT capabilities described in Table 1 reveals that existing literature examines IT capabilities on different levels (i.e., specific, general, and holistic). On a specific level, scholars define very delimited IT capabilities and examine their effect on $\mathrm{AC}$ and corporate performance [5, 27, 38]. For example, Mikalef and Pateli [38] introduce the "ITenabled sensing capability" that helps to monitor 
Table 1. Overview of existing research on IT capabilities for absorptive capacity

\begin{tabular}{|c|c|c|c|c|c|c|}
\hline Ref. & IT capability & Scope & aq & as & tr & ex \\
\hline$[5]$ & Business analytics capability & specific & & $\mathrm{x}$ & & $\mathrm{x}$ \\
\hline [7] & IS capabilities & general & $\mathrm{x}$ & $\mathrm{x}$ & $\mathrm{x}$ & $\mathrm{x}$ \\
\hline [9] & IT capabilities & general & $\mathrm{x}$ & $\mathrm{x}$ & $\mathrm{x}$ & $\mathrm{x}$ \\
\hline [11] & IT-enabled inter-firm knowledge exploration/exploitation capability & holistic & $\mathrm{x}$ & $\mathrm{x}$ & $\mathrm{x}$ & $\mathrm{x}$ \\
\hline [12] & Knowledge absorption capability & holistic & $\mathrm{x}$ & $\mathrm{x}$ & $\mathrm{x}$ & $\mathrm{x}$ \\
\hline [13] & IT-based knowledge capabilities & general & $\mathrm{x}$ & $\mathrm{x}$ & $\mathrm{x}$ & $\mathrm{x}$ \\
\hline [22] & IT-enabled potential / realized / socializing knowledge capabilities & holistic & $\mathrm{x}$ & $\mathrm{x}$ & $\mathrm{x}$ & $\mathrm{x}$ \\
\hline [27] & IT capabilities for sensemaking, IT-enabled coordination capabilities & specific & $\mathrm{x}$ & $\mathrm{x}$ & & \\
\hline [29] & IT-enabled knowledge capabilities - potential/realized/social integration & holistic & $\mathrm{x}$ & $\mathrm{x}$ & $\mathrm{x}$ & $\mathrm{x}$ \\
\hline [32] & IT-driven potential knowledge / knowledge implementation capability & holistic & $\mathrm{x}$ & $\mathrm{x}$ & $\mathrm{x}$ & $\mathrm{x}$ \\
\hline [34] & IT assimilation, Flexible IT infrastructure & specific & & $\mathrm{x}$ & & $\mathrm{x}$ \\
\hline [38] & IT-enabled sensing capability, IT-enabled coordination capability & specific & $\mathrm{x}$ & & & $\mathrm{x}$ \\
\hline [42] & Information and communication technology capabilities & general & $\mathrm{x}$ & & & $\mathrm{x}$ \\
\hline [46] & Outside-in / Inside-out / Spanning IT capabilities & holistic & $\mathrm{x}$ & $\mathrm{x}$ & $\mathrm{x}$ & $\mathrm{x}$ \\
\hline [47] & Analytical / Relational information processing capability & specific & $\mathrm{x}$ & & & $\mathrm{x}$ \\
\hline [50] & Integrated IS capability, Business IT alignment & general & $\mathrm{x}$ & $\mathrm{x}$ & & $\mathrm{x}$ \\
\hline [54] & Business analytics capability & specific & & & & $\mathrm{x}$ \\
\hline [58] & External / Internal IT capability & general & $\mathrm{x}$ & $\mathrm{x}$ & $\mathrm{x}$ & $\mathrm{x}$ \\
\hline 1 & Reference (Ref.), Acquisition (aq), Assin & Sum & 15 & 14 & 10 & 17 \\
\hline
\end{tabular}

competitors or receive customer feedback as a basis for management decisions. These specific definitions allow an exact classification into an AC dimension. On the general level, scholars define IT capabilities very broadly and inexactly, which is why it is impossible to specifically classify them into one AC dimension [7, $8,49]$. Generally defined capabilities have a reference to AC roughly covering all AC dimensions, but do not go very deeply into the description of the underlying IT capabilities. For example, Cepeda-Carrion et al. [7] define the "IS capability" as a capability for classifying and accessing what has been learned and applied. In this respect, technology may codify, store, and recover crucial information from knowledgeacquiring activities [7] and, thus, can be allocated into all AC dimensions. The holistic level includes all approaches that examine IT capabilities explicitly comprising all $\mathrm{AC}$ dimensions $[11,32,46]$. The IT capabilities falling within this last group cover the entire $\mathrm{AC}$ dimensions and describe the impact in a deep relation to the specific AC dimension. Roberts et al. [46], for instance, define outside-in (i.e., acquisition), spanning (i.e., assimilation/ transformation) and inside-out (i.e., exploitation) capabilities. In contrast to the general capabilities, the holistic level comprises detailed capabilities clearly matching the respective $\mathrm{AC}$ dimension.

In summary, scholars describe IT capabilities in the context of AC heterogeneously, often abstractly, and superficially. Thus, a common conceptualization is missing and prevents scholars from deeply analyzing IT capabilities and hindrances toward integrating rising technologies, e.g., AI. Further, this status quo lacks guidance for practitioners and, thus, prevents clearly developing the capabilities required to enhance a company's AC.

\section{DDA capabilities}

Since our goal is to inform the development and implementation of DDAs, we aim for the functional representation of DDAs' capabilities that can be used to represent a DDA as a set of modularized capabilities. Each of the derived DDA capabilities shall then allow an analysis of its functionality individually or in interaction with another capability. Moreover, we emphasize the importance of distinguishing data, information, and knowledge conceptually to integrate knowledge-driven theories with DDAs. Therefore, we follow the idea of Spiegler [51] and consider processes of transformation, following a path from data to information and knowledge. Following existing conceptualizations, we consider data as raw facts and information as organized data [e.g., 37]. According to Alavi and Leidner [3], knowledge differentiates from data or information as it needs to be "possessed in the mind of individuals". Moreover, data does not constitute an immediate value, but carries the potential to be exploited as knowledge-based actions [26]. Knowledge management aims to add actionable value 
to information [40]. To avoid misunderstandings with existing literature not differentiating between data, information, and knowledge, we refer to potential knowledge as knowledge embedded in data. However, potential knowledge is hypothetical since it has not yet been interpreted by the individual. By introducing functional patterns, we follow two goals: First, a functional representation allows us to communicate our concepts unambiguously and, therefore, avoids misunderstanding. Second, a functional representation facilitates arranging DDA capabilities sequentially to represent a DDA. This allows practitioners to, for example, reverse-engineer an existing DDA or to conceptually develop a new DDA. Therefore, we introduce the following principles to approach a functional representation of DDA capabilities: (a) A function $f$ expresses a DDA capability that transforms an input to an output. (b) Inputs and outputs of a function $f$ are either data, information, or knowledge. (c) Data, information, and knowledge are objects that occur in a locality or with a person. (d) Knowledge can be contained in data or information.

In Table 2, we summarize our findings. To foster comprehensiveness, we arranged the DDA capabilities in classes (i.e., create, transform, and exchange). In the following sections, we describe each DDA capability class (i.e., create, transform, and exchange) and its associated capabilities in more detail. Further, in Table 2, we match DDA capabilities with AC dimensions, which we discuss in Section 6.

\subsection{Create}

The capabilities for creating a suitable database focus on making data available as well as on preparing the received data as a basis for further processing. Hence, DDA capabilities allow an organization to make appropriate data accessible internally without yet transforming the data into information or knowledge. Within this capability class, we introduce the DDA capabilities to memorize and prepare.

Memorizing comprises the capability to collect and store data from the real world or external data storage. Considering Alavi and Leidner [3], the role of IT involves gathering and storing potential knowledge. Corresponding capabilities assist companies in collecting potential knowledge from a large number of sources [12]. However, we restrict memorizing to data and information, since knowledge is subject to an agent that interprets it [3]. Besides capturing external data from data storage, DDA may allow a company to digitize real-world observations. For instance, Balasubramanian and Nochur [6] describe the ability of workflow management systems to bring data about events into the system while the process is running regularly. Hence, DDA could support employees conducting their tasks and capturing usage data that may contain relevant knowledge [39]. Some scholars underline the importance of getting virtually in touch and interacting with external actors as a basis for new knowledge and to gain deep insights [22, 58]. After collecting data or information, a company should be able to store various forms of data and information compatibly [35]. Thereby, DDAs include, among others, large-scale storage facilities such as data warehouses [44], document and content management systems [45]. Regardless of the specific memory system, scholars underline the benefits of integrating several information sources into one interface [45] and organizing or codifying it [3, 39].

Preparing refers to a company's capability to process data into the right format and quality. Companies may require this capability since acquired data may only be available in incompatible forms and formats [29]. Although existing research on AC or knowledge management does not directly address this capability, we explicitly include it in order to fully describe the functionality of DDAs. And the importance of data compatibility has been mounting since the variety of data formats has increased with the growth of virtually unlimited different sources of data [23]. Thus, with the emergence of big data, analyzable data requires a transformation of original data into a form suitable for analysis [23]. Accordingly, DDAs' preparing capabilities pre-process data as a prerequisite step for analysis and further processing. Analog to Hashem et al. [23], to prepare data, one needs to take into account whether the data are structured or unstructured. The prepared data are of a high quality, if new data from various sources are compatible with other resources [23]. Not least, data preparation is one step in the established CrossIndustry Standard Process for Data Mining [57].

\subsection{Transform}

The transforming capabilities describe a company's capability to transition between data, information, and knowledge. Thereby, we explicitly distinguish transformations from data to information and from information to knowledge, although existing research often lacks this differentiation (see Section 5). In general, DDAs can enhance the extraction of potential knowledge quickly and accurately [12, 29]. For instance, Ragab and Arisha [45] state that data mining and visualization could be used to generate knowledge from existing raw data. However, since knowledge relies on information, this statement also implies that data mining and visualization could be used to generate information from data. To avoid this 
vagueness, we introduce combining, perceiving, and interpreting capabilities as distinct DDA capabilities.

Considering combining capabilities, we refer to a company's capability to synthesize and merge existing and new data or information [22, 29, 32]. Scholars identified IT as an enabler to recombine heterogeneous potential knowledge, which can increase innovative outcomes [12]. Hence, the role of DDAs can be to link knowledge sources and, hence, to provide wider breadth and depth of knowledge flows [3]. According to Balasubramanian and Nochur [6], activities such as chunking, indexing, filtering, and linking result in a classification scheme and the integration of new information. Combining capabilities may be included in business intelligence tools [29], knowledge repositories [12], or databases and data warehouses [32]. Besides, DDAs could reduce complexity by visualizing interrelations [21] or associating potential knowledge in knowledge maps [45, 54]. Hence, sophisticated data structuring, indexing, and tagging techniques help to navigate through the data jungle $[12,29]$.

With perceiving capabilities, we describe a DDA's capabilities to extract information from data. Appropriate IT capabilities help to extract useful potential knowledge from a wide variety of resources with high speed and accuracy [29]. Accordingly, DDA's perceiving capabilities process data to provide information for the intended recipient [30]. Scholars describe capabilities for extracting potential knowledge that is critical for business [12, 29]. Although IT can foster the transformation from data to information, the involvement of humans can nonetheless be helpful [20]. Hofmann et al. [25], for example, use machine learning to calculate the relatedness of technologies by integrating a large corpus of patent and scientific texts.

When considering interpreting capabilities, we refer to a company's capability to gain knowledge from information. In this context, interpreting capabilities should support an employee in the "process of reflection, enlightenment, or learning" [3]. Hence, companies require capabilities to analyze extracted information facilitating conclusions [5, 47]. DDA, such as data mining applications, can encourage the creation of new knowledge by, among others, organizing, rearranging, or processing information [35]. Accordingly, data mining and visualization could be a means of knowledge discovery [29, 45]. For instance, business analytics tools provide a number of analytic techniques such as descriptive, predictive, and prescriptive analytics [5, 54]. Besides, visualization techniques have a positive effect on understanding the underlying database and, thus, contribute crucially to the process of gaining in-depth insights [22, 29].

\subsection{Exchange}

This capabilities class describes all capabilities supporting the change of data's or information's locality. Accordingly, sharing data or information fosters economic reuse [1]. Besides person-to-person communication, providing access to information could also approach exchange capabilities [60]. Consequently, people could gain access to information by both push and pull mechanisms [6]. To account for accessing information, we introduce searching (i.e., pull) as well as sharing capabilities (i.e., push).

Table 2. Data-driven application capabilities

\begin{tabular}{|c|c|c|c|c|c|c|c|}
\hline & Capability & Description & Functional patterns & aq & as & $\operatorname{tr}$ & ex \\
\hline \multirow{2}{*}{ 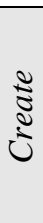 } & Memorizing & DDA's capability to collect and store data & $\begin{array}{c}f(\text { real world })=D^{\text {int. }} \\
f\left(D^{\text {ext. }}\right)=D^{\text {int. }} \\
f\left(I^{\text {ext. }}\right)=I^{\text {int. }}\end{array}$ & $\mathrm{x}$ & & & \\
\hline & Preparing & $\begin{array}{l}\text { DDA's capability to pre-process data regarding } \\
\text { data format or quality }\end{array}$ & $f\left(D_{A}\right)=D_{A}{ }^{\prime}$ & & $\mathrm{x}$ & & \\
\hline \multirow{3}{*}{$\begin{array}{l}\Sigma \\
\vdots \\
\vdots \\
\vdots \\
\vdots \\
\vdots\end{array}$} & Perceiving & DDA's capability to extract information from data & $f\left(D_{A}\right)=I_{A}$ & & $\mathrm{x}$ & & \\
\hline & Combining & $\begin{array}{l}\text { DDA's capability to synthesize and merge new } \\
\text { data or information with existing information }\end{array}$ & $\begin{aligned} f\left(D_{A}, I_{B}\right) & =I_{A B} \\
f\left(I_{A}, I_{B}\right) & =I_{A B}\end{aligned}$ & & $\mathrm{x}$ & $\mathrm{x}$ & \\
\hline & Interpreting & $\begin{array}{l}\text { DDA's capability to gain knowledge from } \\
\text { information }\end{array}$ & $f(I)=K$ & & $\mathrm{x}$ & $\mathrm{x}$ & $\mathrm{x}$ \\
\hline \multirow{2}{*}{ 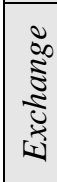 } & Searching & $\begin{array}{l}\text { DDA's capability to identify data or information } \\
\text { relevant for the user }\end{array}$ & $\begin{aligned} f\left(D_{A}\right) & =D_{a} ; \quad \text { with } a \in A \\
f\left(I_{A}\right) & =I_{a} ; \quad \text { with } a \in A\end{aligned}$ & $\mathrm{x}$ & & & $\mathrm{x}$ \\
\hline & Sharing & $\begin{array}{l}\text { DDA's capability to disseminate and embed } \\
\text { information }\end{array}$ & $\begin{aligned} f\left(D^{A}\right) & =D^{B} \\
f\left(I^{A}\right) & =I^{B}\end{aligned}$ & & $\mathrm{x}$ & & $\mathrm{x}$ \\
\hline
\end{tabular}

Legend: Data (D), Information (I), Knowledge (K), External (ext.), Internal (int.),

Acquisition (aq), Assimilation (as), Transformation (tr), Exploitation (ex) 
Thereby, the exchanged object (i.e., data or information) should follow the idea of reusability so that it can be used in other settings without losing its comprehensiveness [6]. Similar to the capabilities already introduced, most papers do not distinguish between the type of exchanged objects. However, we argue that a DDA could only transfer unstructured data or organized data (i.e., information) but no knowledge. Nonetheless, scholars attribute great importance to providing access to potential knowledge [17].

A substantial capability in the context of AC is to search for potential knowledge from various external sources $[12,29,58]$. Thereby, IT can enhance the identification of potential knowledge [12, 29] by locating data or information with search and retrieval mechanisms [3]. Joshi et al. [29] refer to IT that enhances a company's capability to retrieve useful knowledge quickly and accurately. Thereby, DDA can support this process in different ways. First, retrieval techniques such as query software and search engines [29] can independently search. Second, web mining techniques [12] enable a less rigid search. Third, knowledge representations such as ontologies [39] guide the user in its search process. DDA capabilities can support not only the internal search for data or information but also the external search. For instance, companies may rely on search engines such as Google or forums to access a larger body of information [21]. Searching activities may not only take place once, but can also be continuous. Searching could also refer to continuously monitoring. For example, some scholars refer to sensing and monitoring capabilities supporting the observation of competitor and customer behavior and, thus, identify potential knowledge [38, 47].

Sharing capabilities facilitate data or information transfer. Herewith, sharing is the most frequently mentioned dimension in knowledge management frameworks [24]. Recommender systems could disseminate information in a personalized manner by anticipating the interests of a user [52]. Moreover, information systems relying on codified knowledge, such as workflow systems or expert systems, could also indirectly foster an organization's sharing capability [56]. Further, AC literature mentions IT components should be connected in order to facilitate integrated data sources within and across organizational boundaries [34]. This IT connectivity enables a company to transfer potential knowledge across functional units [34]. But effective change and knowledge integration require interaction among individuals [58] and across different units or functions $[38,50]$. IT systems enable coordination and foster communication [7, 58], thus, reducing cognitive, structural, and political barriers [50], e.g., by corporate social media.

\section{The effect of DDA capabilities on AC}

In the acquisition dimension, companies identify and acquire possibly relevant knowledge [59]. Corresponding IT capabilities can enhance intensity and speed [12, 29]. Searching capabilities enable scanning through massive amounts of data in a short time, locating useful data. Scholars describe this capability by the respective software for potential knowledge location [22] from a wide variety of resources [29]. Memorizing capabilities expand the utilization of (external) data and, that way, increase the opportunity to acquire additional potential external knowledge [58]. Additionally, knowledge acquisition comprises the step to obtain the potential knowledge that is critical to the operations of a company [29]. Thus, the identified external data is collected and firstly stored through memorizing capabilities, which prepare the data basis for further analysis.

In the assimilation dimension, companies evaluate the potential knowledge in order to entirely comprehend it in the context of what organizational members already know [59]. In a first step, preparing capabilities support the processing of the obtained data to ensure the data quality and compatibility. In literature, IT is described as a capability that can help to effectively store potential knowledge [3] and allow employees to interpret it in a consistent manner [29]. But the comprehension of potential knowledge is dependent on prior related knowledge [59]. Hence, a consolidation with current explicit knowledge under consideration of combining capabilities is obligatory and sustainably connects data or information with existing information. Cui et al. [12] also refer to knowledge repositories that facilitate the capability to recombine potential knowledge. Moreover, in this AC dimension, perceiving capabilities ease the extraction of hidden data and, thus, enhance the available dataset or information basis, while interpreting capabilities enable preliminary assessments and visualizations. Therefore, scholars identified business analytics to prepare an appropriate context to draw conclusions [5]. In some instances, comprehension of potential knowledge is dependent on complementary assets [59]. Thus, sharing capabilities allow a company to involve subject matter experts and, thus, improve the ability to understand potential knowledge.

In the transformation dimension, companies transform and associate the new potential knowledge with the existing knowledge to develop and refine the required routines [59]. Combining capabilities support this process of transformation by synthesizing, developing, adding, reconfiguring, or deleting absorbed potential knowledge [29]. But transformation is particularly difficult when the 
existing knowledge and the new potential knowledge reflect incompatible frames of reference [59]. Therefore, interpreting capabilities provide capabilities to visualize and analyze extracted information and, thus, support transformation [29].

In the exploitation dimension, companies utilize the newly gained potential knowledge by refining, extending, or leveraging existing corporate capabilities [59]. Capabilities to access potential knowledge facilitate the incorporation of knowledge into a company's operations [59]. IT can support access by searching and interpreting capabilities providing access to data and information, but also enable new perspectives through different reports and visualizations [32] as a basis for the manifold application. Finally, sharing capabilities foster communication and the transfer of potential knowledge across different functions and boundaries, thus facilitating the necessary connectedness of data and information among individuals across organizational borders $[7,58]$.

\section{Conclusion}

The use of data is a relevant source for gaining competitive advantage and, thus, companies increasingly use data to guide decision-making and improve business processes [19]. In this paper, we have investigated which capabilities can be assigned to DDAs and how these capabilities can foster an organization's AC. In doing so, we derived seven DDA capabilities and mapped them into the AC dimensions. Our results reveal valuable insights: First, DDA offers several distinct capabilities specifically fostering AC dimensions. Thereby, interpreting capabilities play an important role, since they are relevant in a total of three dimensions. Second, existing DDAs often comprise more than one capability. Third, there are often different ways to approach a DDA capability. Thus, this work provides added value for the scientific discourse and to practice.

As a fundamental theoretical contribution, we bring together the literature streams of IT capabilities and AC systematically. We elaborate on how the existing literature describes IT capabilities for AC and, hence, provide a structured literature review on the impact of IT for AC (see Section 4). By matching the relevant literature of IT capabilities and their influence on $\mathrm{AC}$, we make an important contribution to the research of the digital transformation of companies and enable a systematic consideration for the development and introduction of DDAs in businesses.

With the functional representation of DDA capabilities, we create a clear conceptual basis for the exploration of DDA capabilities, their connection with other forms of capabilities, and their influence on AC. Thus, we introduce DDA capabilities in a functional representation that divided a DDA's functionality into separate and analyzable functions. Hence, the theoretically-based and modularized DDA capabilities represent a conceptual basis for further research.

By operationalizing the DDA capabilities, we make an important contribution to practice. The modularized DDA capabilities approach provides a valuable guide for the development, selection, and implementation of DDA in enterprises. It informs practitioners with a clear distinction of capabilities and enables the systematic development of AC.

Despite the valuable contribution of our proposed conceptualization of DDA capabilities, it is also subject to some limitations. As our elaboration of the DDA capabilities is based on an extensive literature search and is thus a conceptual approach, in a next step, we will empirically evaluate the results in further research. Also, we will use our DDA capabilities to analyze technology trends (e.g., AI) against the background of AC. In addition, future research may rely on the conceptualization of the DDA capabilities to analyze specific capabilities or their interplay.

\section{References}

[1] Abbaraju, S.C., S.R. Karangutkar, V.S. Deshpande, and A.M. Deshmukh, "A Study on Knowledge Management Practices in Indian IT Industry", ITIHAS - The Journal of Indian Management, 6(2), 2016, pp. 22-29.

[2] Ahmed, A., N. Masri, Y.A. Sultan, A.N. Akkila, A. Almasri, A.Y. Mahmoud, I. Zaqout, and S.S. Abu-Naser, "Knowledge-Based Systems Survey", International Journal of Academic Engineering Research (IJAER), 3(7), 2019, pp. 1-22.

[3] Alavi, M. and D.E. Leidner, "Review: Knowledge Management and Knowledge Management Systems: Conceptual Foundations and Research Issues", MIS Quarterly, 25(1), 2001, pp. 107-136.

[4] Alter, S., "Defining information systems as work systems: implications for the IS field", European Journal of Information Systems, 17(5), 2008, pp. 448-469.

[5] Ashrafi, A., A.Z. Ravasan, P. Trkman, and S. Afshari, "The role of business analytics capabilities in bolstering firms' agility and performance", International Journal of Information Management, 47, 2019, pp. 1-15.

[6] Balasubramanian, P. and K. Nochur, "Managing process knowledge for decision support", Decision Support Systems, 27(1/2), 1999, p. 145.

[7] Cepeda-Carrion, G., J.G. Cegarra-Navarro, and D. Jimenez-Jimenez, "The Effect of Absorptive Capacity on Innovativeness: Context and Information Systems 
Capability as Catalysts", British Journal of Management, 23(1), 2012, pp. 110-129.

[8] Choi, S., "Developing relationship-specific memory and absorptive capacity in interorganizational relationships", Information Technology and Management, 15(4), 2014, pp. 223-238.

[9] Chou, S.W., "Knowledge creation: absorptive capacity, organizational mechanisms, and knowledge storage/retrieval capabilities", Journal of Information Science, 31(6), 2005, pp. 453-465.

[10] Cohen, W.M. and D.A. Levinthal, "Absorptive Capacity: A New Perspective on Learning and Innovation", Administrative Science Quarterly, 35(1), 1990, p. 128.

[11] Cui, T., Y. Tong, H.-H. Teo, and J. Li, "Managing Knowledge Distance: IT-Enabled Inter-Firm Knowledge Capabilities in Collaborative Innovation", Journal of Management Information Systems, 37(1), 2020, pp. 217250.

[12] Cui, T., Y. Wu, and Y. Tong, "Exploring ideation and implementation openness in open innovation projects: ITenabled absorptive capacity perspective", Information \& Management, 55(5), 2018, pp. 576-587.

[13] Datta, A., "IT-Based Knowledge Capability and Commercialization of Innovations: Modeling the Impacts of Ambidexterity and Absorptive Capacity", International Journal of Knowledge Management, 8(3), 2012, pp. 83-97.

[14] Erickson, S. and H. Rothberg, "Big Data and Knowledge Management: Establishing a Conceptual Foundation", Electronic Journal of Knowledge Management, 2(12), 2017, pp. 108-116.

[15] Feng, W., Y. Duan, Z. Fu, and B. Mathews, "Understanding expert systems applications from a knowledge transfer perspective", Knowledge Management Research \& Practice, 7(2), 2009, pp. 131-141.

[16] Fowler, A., "The role of AI-based technology in support of the knowledge management value activity cycle", The Journal of Strategic Information Systems, 9(23), 2000, pp. 107-128.

[17] Gonzalez, L.M., R.E. Giachetti, and G. Ramirez, "Knowledge management-centric help desk: specification and performance evaluation", Decision Support Systems, 40(2), 2005, pp. 389-405.

[18] Grant, R.M., "Toward a knowledge-based theory of the firm", Strategic Management Journal, 17(S2), 1996, pp. 109-122.

[19] Grover, V., R.H.L. Chiang, T.-P. Liang, and D. Zhang, "Creating Strategic Business Value from Big Data Analytics: A Research Framework", Journal of Management Information Systems, 35(2), 2018, pp. 388423.

[20] Grover, V. and T.H. Davenport, "General perspectives on knowledge management: Fostering a research agenda", Journal of Management Information Systems, 18(1), 2001, pp. 5-21.
[21] Haamann, T. and D. Basten, "The role of information technology in bridging the knowing-doing gap: an exploratory case study on knowledge application", Journal of Knowledge Management, 23(4), 2019, pp. 705-741.

[22] Han, S., A. Datta, K.D. Joshi, and L. Chi, "Innovation Through Boundary Spanning: The Role of IT in Enabling Knowledge Flows Across Technological and Geographical Boundaries", International Journal of Knowledge Management, 13(4), 2017, pp. 90-110.

[23] Hashem, I.A.T., I. Yaqoob, N.B. Anuar, S. Mokhtar, A. Gani, and S. Ullah Khan, "The rise of "big data" on cloud computing: Review and open research issues", Information Systems, 47, 2015, pp. 98-115.

[24] Heisig, P., "Harmonisation of knowledge management - comparing $160 \mathrm{KM}$ frameworks around the globe", Journal of Knowledge Management, 13(4), 2009, pp. 4-31.

[25] Hofmann, P., R. Keller, and N. Urbach, "Intertechnology relationship networks: Arranging technologies through text mining", Technological Forecasting and Social Change, 143, 2019, pp. 202-213.

[26] Houari, N. and B.H. Far, "Application of intelligent agent technology for knowledge management integration", in Proceedings of the Third IEEE International Conference on Cognitive Informatics, ICCI 2004, C. Chan, Editor. 2004. IEEE Computer Society: Los Alamitos, Calif.

[27] Im, G. and A. Rai, "IT-Enabled Coordination for Ambidextrous Interorganizational Relationships", Information Systems Research, 25(1), 2014, pp. 72-92.

[28] Iyengar, K., J.R. Sweeney, and R. Montealegre, "Information technology use as a learning mechanism: the impact of it use on knowledge transfer effectiveness, absorptive capacity, and franchisee performance", MIS Quarterly, 39(3), 2015, 615-642.

[29] Joshi, K.D., L. Chi, A. Datta, and S. Han, "Changing the Competitive Landscape: Continuous Innovation Through IT-Enabled Knowledge Capabilities", Information Systems Research, 21(3), 2010, pp. 472-495.

[30] Kusumawardhani, M. and T. Markeset, "Asset Integrity Knowledge Management: A Case Study from the Petroleum Industry", Operations and Supply Chain Management, 8(3), 2015, pp. 146-153.

[31] Lane, P.J., B.R. Koka, and S. Pathak, "The Reification of Absorptive Capacity: A Critical Review and Rejuvenation of the Construct", Academy of Management Review, 31(4), 2006, pp. 833-863.

[32] Li, H., Q. Zhang, and Z. Zheng, "A Mediating Effect of Knowledge Integration and Moderating Effect of Organizational Task Environment", Enterprise Information Systems, 13(9), 2019, pp. 1323-1346.

[33] Liebowitz, J., "Knowledge management and its link to artifcial intelligence", Expert Systems with Applications, 20, 2001, pp. 1-6.

[34] Liu, H., W. Ke, K.K. Wei, and Z. Hua, "The impact of IT capabilities on firm performance: The mediating roles of 
absorptive capacity and supply chain agility", Decision Support Systems, 54(3), 2013, pp. 1452-1462.

[35] Malhotra, A., S. Gosain, and O.A. El Sawy, "Absorptive capacity configurations in supply chains: Gearing for partner-enabled market knowledge creation", MIS Quarterly, 29(1), 2005, pp. 145-187.

[36] Mao, H., S. Liu, J. Zhang, and Y. Zhang, "Information technology competency and organizational agility: Roles of absorptive capacity and information intensity", in Proceedings of the 25th European Conference on Information Systems (ECIS). 2017.

[37] Metaxiotis, K., K. Ergazakis, E. Samouilidis, and J. Psarras, "Decision support through knowledge management: the role of the artificial intelligence", Information Management \& Computer Security, 11(5), 2003, pp. 216-221.

[38] Mikalef, P. and A. Pateli, "Information technologyenabled dynamic capabilities and their indirect effect on competitive performance: Findings from PLS-SEM and fsQCA", Journal of Business Research, 70, 2017, pp. 1-16.

[39] Milton, N., N. Shadbolt, H. Cottam, and M. Hammersley, "Towards a knowledge technology for knowledge management", International Journal of HumanComputer Studies, 51(3), 1999, pp. 615-641.

[40] Nemati, H.R., D.M. Steiger, L.S. Iyer, and R.T. Herschel, "Knowledge warehouse: an architectural integration of knowledge management, decision support, artificial intelligence and data warehousing", Decision Support Systems, 33(2), 2002, pp. 143-161.

[41] Nonaka, I. and H. Takeuchi, The knowledge creating company: How Japanese companies create the dynamics of innovation, Oxford Univ. Press, New York, 1995.

[42] Parida, V., P. Oghazi, and S. Cedergren, "A study of how ICT capabilities can influence dynamic capabilities", Journal of Enterprise Information Management, 29(2), 2016, pp. 179-201.

[43] Pavlou, P.A. and O.A. El Sawy, "From IT Leveraging Competence to Competitive Advantage in Turbulent Environments: The Case of New Product Development", Information Systems Research, 17(3), 2006, pp. 198-227.

[44] Qi, J., L.D. Xu, H.Y. Shu, and H.Z. Li, "Knowledge management in OSS - an enterprise information system for the telecommunications industry", Systems Research and Behavioral Science, 23(2), 2006, pp. 177-190.

[45] Ragab, M.A.F. and A. Arisha, "Knowledge management and measurement: a critical review", Journal of Knowledge Management, 17(6), 2013, pp. 873-901.

[46] Roberts, N., P.S. Galluch, M. Dinger, and V. Grover, "Absorptive Capacity and Information Systems Research: Review, Synthesis, and Directions for Future Research", MIS Quarterly, 36(2), 2012, pp. 625-648.

[47] Saldanha, T.J.V., S. Mithas, and M.S. Krishnan, "Leveraging Customer Involvement for Fueling Innovation: The Role of Relational and Analytical Information
Processing Capabilities", MIS Quarterly, 41(1), 2017, 267286.

[48] Sambamurthy, V. and M. Subramani, "Special Issue on information technologies and knowledge management", MIS Quarterly, 29(2), 2005.

[49] Saraf, N., C.S. Langdon, and S. Gosain, "IS application capabilities and relational value in interfirm partnerships", Information Systems Research, 18(3), 2007, pp. 320-339.

[50] Setia, P. and P.C. Patel, "How information systems help create OM capabilities: Consequents and antecedents of operational absorptive capacity", Journal of Operations Management, 31(6, SI), 2013, pp. 409-431.

[51] Spiegler, I., "Information systems: a construct of transformations", IEEE Transactions on Systems, Man, and Cybernetics, 25(7), 1995, pp. 1121-1129.

[52] Stenmark, D., "Leveraging tacit organizational knowledge", Journal of Management Information Systems, 17(3), 2000, pp. 9-24.

[53] Tsui, E., B.J. Garner, and S. Staab, "The role of Artificial Intelligence in Knowledge Management", Knowledge based systems, 13(5), 2000, pp. 235-239.

[54] Wang, Y. and T.A. Byrd, "Business analytics-enabled decision-making effectiveness through knowledge absorptive capacity in health care", Journal of Knowledge Management, 21(3), 2017, pp. 517-539.

[55] Webster, J. and R.T. Watson, "Analyzing the Past to Prepare for the Future: Writing a Literature Review", MIS Quarterly, 26(2), 2002, pp. xiii-xxiii.

[56] Wills, M.J., S. Sarnikar, O.F. El-Gayar, and A.V. Deokar, "Information Systems and Healthcare XXXIV: Clinical Knowledge Management Systems - Literature Review and Research Issues for Information Systems", Communications of the Association for Information Systems, 26(1), 2010, pp. 565-598.

[57] Wirth, R. and J. Hipp, "CRISP-DM: Towards a standard process model for data mining", in Proceedings of the 4th international conference on the practical applications of knowledge discovery and data mining. 2000. Springer-Verlag: London, UK.

[58] Wu, S., X. Ding, R. Liu, and H. Gao, "How does IT capability affect open innovation performance? The mediating effect of absorptive capacity", European Journal of Innovation Management, ahead-of-print, 2019.

[59] Zahra, S.A. and G. George, "The Net-Enabled Business Innovation Cycle and the Evolution of Dynamic Capabilities", Information Systems Research, 13(2), 2002, pp. 147-150.

[60] Zhang, M.J., "An Empirical Assessment of the Performance Impacts of IS Support for Knowledge Transfer", International Journal of Knowledge Management, 3(1), 2007, pp. 66-85. 